\title{
Rigidity and flatness of the image of certain classes of mappings having tangential laplacian
}

Article

Accepted Version

Abugirda, H., Ayanbayev, B. and Katzourakis, N. (2020) Rigidity and flatness of the image of certain classes of mappings having tangential laplacian. Rocky Mountain Journal of Mathematics, 50 (2). pp. 383-396. ISSN 0035-7596 doi: https://doi.org/10.1216/rmj.2020.50.383 Available at https://centaur.reading.ac.uk/95576/

It is advisable to refer to the publisher's version if you intend to cite from the work. See Guidance on citing.

To link to this article DOI: http://dx.doi.org/10.1216/rmj.2020.50.383

Publisher: Rocky Mountain Mathematics Consortium

All outputs in CentAUR are protected by Intellectual Property Rights law, including copyright law. Copyright and IPR is retained by the creators or other copyright holders. Terms and conditions for use of this material are defined in the End User Agreement.

www.reading.ac.uk/centaur 
Central Archive at the University of Reading

Reading's research outputs online 


\title{
RIGIDITY AND FLATNESS OF THE IMAGE OF CERTAIN CLASSES OF MAPPINGS HAVING TANGENTIAL LAPLACIAN
}

\author{
Hussien Abugirda, Birzhan Ayanbayev and Nikos Katzourakis
}

This paper is dedicated to Gunnar Aronsson with the utmost esteem for his pioneering work.

In this paper we consider the PDE system of vanishing normal projection of the Laplacian for $C^{2}$ maps $u: \mathbb{R}^{n} \supseteq \Omega \rightarrow \mathbb{R}^{N}:$

$$
\llbracket \mathrm{D} u \rrbracket^{\perp} \Delta u=0 \quad \text { in } \Omega .
$$

This system has discontinuous coefficients and geometrically expresses the fact that the Laplacian is a vector field tangential to the image of the mapping. It arises as a constituent component of the $p$-Laplace system for all $p \in[2, \infty]$. For $p=\infty$, the $\infty$-Laplace system is the archetypal equation describing extrema of supremal functionals in vectorial calculus of variations in $L^{\infty}$. Herein we show that the image of a solution $u$ is piecewise affine if either the rank of $\mathrm{D} u$ is equal to one or $n=2$ and $u$ has additively separated form. As a consequence we obtain corresponding flatness results for $p$-Harmonic maps for $p \in[2, \infty]$.

\section{Introduction}

Suppose that $n, N$ are integers and $\Omega$ an open subset of $\mathbb{R}^{n}$. In this paper we study geometric aspects of the image $u(\Omega) \subseteq \mathbb{R}^{N}$ of certain classes of $C^{2}$ vectorial solutions $u: \mathbb{R}^{n} \supseteq \Omega \rightarrow \mathbb{R}^{N}$ to the following nonlinear degenerate elliptic PDE system:

$$
\llbracket \mathrm{D} u \rrbracket^{\perp} \Delta u=0 \quad \text { in } \Omega .
$$

Here, for the map $u$ with components $\left(u_{1}, \ldots, u_{N}\right)^{\top}$ the notation $\mathrm{D} u$ symbolises the gradient matrix

$$
\mathrm{D} u(x)=\left(\mathrm{D}_{i} u_{\alpha}(x)\right)_{i=1, \ldots, n}^{\alpha=1, \ldots, N} \in \mathbb{R}^{N \times n}, \quad \mathrm{D}_{i} \equiv \partial / \partial x_{i},
$$

$\Delta u$ stands for the Laplacian

$$
\Delta u(x)=\sum_{i=1}^{n} \mathrm{D}_{i i}^{2} u(x) \in \mathbb{R}^{N}
$$

Katzourakis is the corresponding author. He has been partially financially supported through the EPSRC grant EP/N017412/1. 2010 AMS Mathematics subject classification: 35B06, 35B65, 35D99, 49N60, 49 N99.

Keywords and phrases: vectorial calculus of variations, calculus of variations in $L^{\infty}, \infty$-Laplacian, $p$-Laplacian, rank-one solutions, special separated solutions, rigidity, flatness.

Received by the editors on December 30, 2018, and in revised form on August 10, 2019. 
and for any $X \in \mathbb{R}^{N \times n}, \llbracket X \rrbracket^{\perp}$ denotes the orthogonal projection on the orthogonal complement of the range of linear map $X: \mathbb{R}^{n} \rightarrow \mathbb{R}^{N}$ :

$$
\llbracket X \rrbracket^{\perp}:=\operatorname{Proj}_{\mathrm{R}(X)^{\perp}} .
$$

Our general notation will be either self-explanatory, or otherwise standard as, e.g., in [14; 12]. Note that, since the rank is a discontinuous function, the map $\mathbb{[} \cdot \mathbb{1}^{\perp}$ is discontinuous on $\mathbb{R}^{N \times n}$; therefore, the PDE system (1-1) has discontinuous coefficients. The geometric meaning of (1-1) is that the Laplacian vector field $\Delta u$ is tangential to the image $u(\Omega)$ and hence (1-1) is equivalent to the next statement: there exists a vector field

$$
\mathrm{A}: \mathbb{R}^{n} \supseteq \Omega \rightarrow \mathbb{R}^{n}
$$

such that

$$
\Delta u=\mathrm{D} u \mathrm{~A} \quad \text { in } \Omega .
$$

As we show later, the vector field is generally discontinuous (Lemma 6).

Our interest in (1-1) stems from the fact that it is a constituent component of the $p$-Laplace PDE system for all $p \in[2, \infty]$. Further, contrary perhaps to appearances, (1-1) is in itself a variational PDE system but in a nonobvious way. Deferring temporarily the specifics of how exactly (1-1) arises and what is the variational principle associated with it, let us recall that, for $p \in[2, \infty)$, the celebrated $p$-Laplacian is the divergence system

$$
\Delta_{p} u:=\operatorname{Div}\left(|\mathrm{D} u|^{p-2} \mathrm{D} u\right)=0 \quad \text { in } \Omega
$$

and comprises the Euler-Lagrange equation which describes extrema of the model $p$-Dirichlet integral functional

$$
E_{p}(u):=\int_{\Omega}|\mathrm{D} u|^{p}, \quad u \in W^{1, p}\left(\Omega, \mathbb{R}^{N}\right),
$$

in conventional vectorial calculus of variations. Above and subsequently, for any $X \in \mathbb{R}^{N \times n}$, the notation $|X|$ symbolises its Euclidean (Frobenius) norm:

$$
|X|=\left(\sum_{\alpha=1}^{N} \sum_{i=1}^{n}\left(\boldsymbol{X}_{\alpha i}\right)^{2}\right)^{1 / 2}
$$

The pair (1-3)-(1-4) is of paramount important in applications and has been studied exhaustively. The extremal case of $p \rightarrow \infty$ in (1-3)-(1-4) is much more modern and intriguing, in that totally new phenomena arise which are not present in the scalar case. It turns out that one then obtains the following nondivergence PDE system

$$
\Delta_{\infty} u:=\left(\mathrm{D} u \otimes \mathrm{D} u+|\mathrm{D} u|^{2} \llbracket \mathrm{D} u \rrbracket^{\perp} \otimes \mathrm{I}\right): \mathrm{D}^{2} u=0 \quad \text { in } \Omega,
$$

which is known as the $\infty$-Laplacian. In index form, (1-5) reads

$$
\sum_{\beta=1}^{N} \sum_{i, j=1}^{n}\left(\mathrm{D}_{i} u_{\alpha} \mathrm{D}_{j} u_{\beta}+|\mathrm{D} u|^{2} \llbracket \mathrm{D} u \rrbracket_{\alpha \beta}^{\perp} \delta_{i j}\right) \mathrm{D}_{i j}^{2} u_{\beta}=0, \quad \alpha=1, \ldots, N .
$$


The system (1-3) plays the role of the Euler-Lagrange equation and arises in connexion with variational problems for the supremal functional

$$
E_{\infty}(u, \mathcal{O}):=\|\mathrm{D} u\|_{L^{\infty}(\mathcal{O})}, \quad u \in W^{1, \infty}\left(\Omega, \mathbb{R}^{N}\right), \mathcal{O} \Subset \Omega .
$$

The scalar case of $N=1$ in (1-5)-(1-6) was pioneered by G. Aronsson in the 1960s [2-6] who initiated the field of calculus of variations in $L^{\infty}$, namely the study of supremal functionals and of their associated equations describing critical points. Since then, the field has developed tremendously and there is extensive relevant literature, some of which is surveyed in [19]. In particular, although vectorial supremal functionals began to be explored early enough, the $\infty$-Laplace system (1-5) which describes the necessary critical conditions in $L^{\infty}$ in the vectorial case $N \geq 2$ first arose in the early 2010s in [15]. The area is now developing very rapidly due to both the mathematical significance as well as the importance for applications in diverse areas like image processing [13;23], quasiconformal mappings and the singular value problem (see $[1 ; 11 ; 24 ; 20]$ and references therein).

In this paper we focus on the $C^{2}$ case and establish the geometric rigidity and flatness of the images of solutions $u: \mathbb{R}^{n} \supseteq \Omega \rightarrow \mathbb{R}^{N}$ to the nonlinear system (1-1), under the assumption that either $\mathrm{D} u$ has rank at most 1 , or that $n=2$ and $u$ has an additively separated form, see (1-7). As a consequence, we obtain corresponding flatness results for the images of solutions to (1-3) and (1-5). Both aforementioned classes of solutions furnish particular examples which provide substantial intuition for the behaviour of general extremal maps in calculus of variations in $L^{\infty}$, see, e.g., $[7 ; 8 ; 10 ; 16 ; 17 ; 19 ; 24]$ where solutions of this form have been studied. Obtaining further information for the still largely mysterious behaviour of $\infty$-Harmonic maps is perhaps the greatest driving force to isolate and study the particular nonlinear system (1-1). For example, it is not yet know to what extend the possible discontinuities of the coefficients relates to the failure of absolute minimality.

It is also worth clarifying that, although as it is well-known the Dirichlet problem over a bounded domain may not in general be solvable for the $\infty$-Laplacian not even in the scalar-valued case, if one does not prescribe boundary values (and we do not in this paper) it can be demonstrated that infinitely many nontrivial classical solutions do exist, in particular of the form arising in this paper (see for instance the explicit constructions of $C^{2}$ solutions in [16]). Therefore, the results herein are not void and numerous solutions as those exhibited herein do exist.

Let us note that the rank-one case includes the scalar and the one-dimensional case (i.e., when $\min \{n, N\}=1$ ), although in the case of $N=1$ (in which the single $\infty$-Laplacian reduces to $\mathrm{D} u \otimes \mathrm{D} u$ : $\left.\mathrm{D}^{2} u=0\right)(1-1)$ has no bearing since it vanishes identically at any noncritical point.

The effect of (1-1) to the flatness of the image can be seen through the $L^{\infty}$ variational principle introduced in [18], wherein it was shown that solutions to (1-1) of constant rank can be characterised as those having minimal area with respect to (1-6)-(1-4). More precisely, therein the following result was proved:

Theorem 1 [18, Theorem 2.7, Lemma 2.2]. Given $N \geq n \geq 1$, let $u: \mathbb{R}^{n} \supseteq \Omega \rightarrow \mathbb{R}^{N}$ be a $C^{2}$ immersion defined on the open set $\Omega$ (more generally $u$ can be a map with constant rank of its gradient on $\Omega$ ). Then, the following statements are equivalent:

(1) The map u solves the PDE system (1-1) on $\Omega$. 


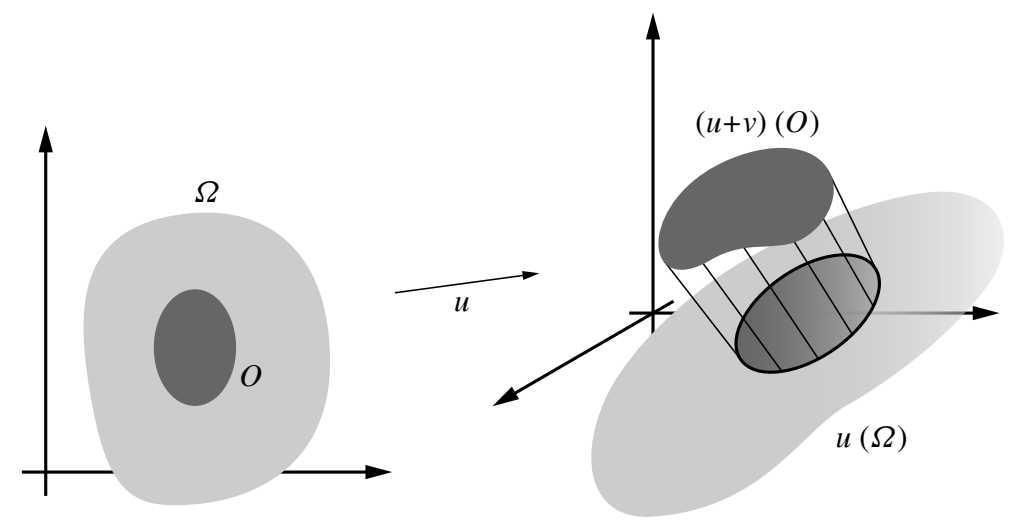

Figure 1. Illustration of the variational principle characterising (1-1).

(2) For all $p \in[2, \infty]$, for all compactly supported domains $\mathcal{O} \Subset \Omega$ and all $C^{1}$ vector fields $v: \mathcal{O} \rightarrow \mathbb{R}^{N}$ which are normal to the image $u(\mathcal{O}) \subseteq \mathbb{R}^{N}$ (without requiring to vanish on $\partial \mathcal{O}$ ), namely those for which $v=\llbracket \mathrm{D} u \rrbracket^{\perp} v$ in $\mathcal{O}$, we have

$$
\|\mathrm{D} u\|_{L^{p}(\mathcal{O})} \leq\|\mathrm{D} u+\mathrm{D} v\|_{L^{p}(\mathcal{O})} .
$$

(3) The same statement as in item (2) holds, but only for some $p \in[2, \infty]$.

If in addition $p<\infty$ in (2)-(3), then we may further restrict the class of normal vector fields to those satisfying $\left.\nu\right|_{\partial \mathcal{O}}=0$ (see Figure 1).

In the paper [18], it was also shown that in the conformal class, (1-1) expresses the vanishing of the mean curvature vector of $u(\Omega)$.

The effect of (1-1) to the flatness of the image can be easily seen in the case of $n=1 \leq N$ as follows: since

and in one dimension we have

$$
\llbracket u^{\prime} \rrbracket^{\perp} u^{\prime \prime}=0 \quad \text { in } \Omega \subseteq \mathbb{R}
$$

$$
\llbracket u^{\prime} \rrbracket^{\perp}= \begin{cases}\mathrm{I}-\frac{u^{\prime} \otimes u^{\prime}}{\left|u^{\prime}\right|^{2}} & \text { on }\left\{u^{\prime} \neq 0\right\}, \\ \mathrm{I} & \text { on }\left\{u^{\prime}=0\right\},\end{cases}
$$

we therefore infer that $u^{\prime \prime}=f u^{\prime}$ on the open set $\left\{u^{\prime} \neq 0\right\} \subseteq \mathbb{R}$ for some function $f$, readily yielding after an integration that $u(\Omega)$ is necessarily contained in a piecewise polygonal line of $\mathbb{R}^{N}$. As a generalisation of this fact, our first main result herein is the following:

Theorem 2 (rigidity and flatness of rank-one maps with tangential Laplacian). Let $\Omega \subseteq \mathbb{R}^{n}$ be an open set and $n, N \geq 1$. Let $u \in C^{2}\left(\Omega, \mathbb{R}^{N}\right)$ be a solution to the nonlinear system (1-1) in $\Omega$, satisfying that the rank of its gradient matrix is at most one:

$$
\operatorname{rk}(\mathrm{D} u) \leq 1 \quad \text { in } \Omega .
$$

Then, its image $u(\Omega)$ is contained in a polygonal line in $\mathbb{R}^{N}$, consisting of an at most countable union of affine straight line segments (possibly with self-intersections). 

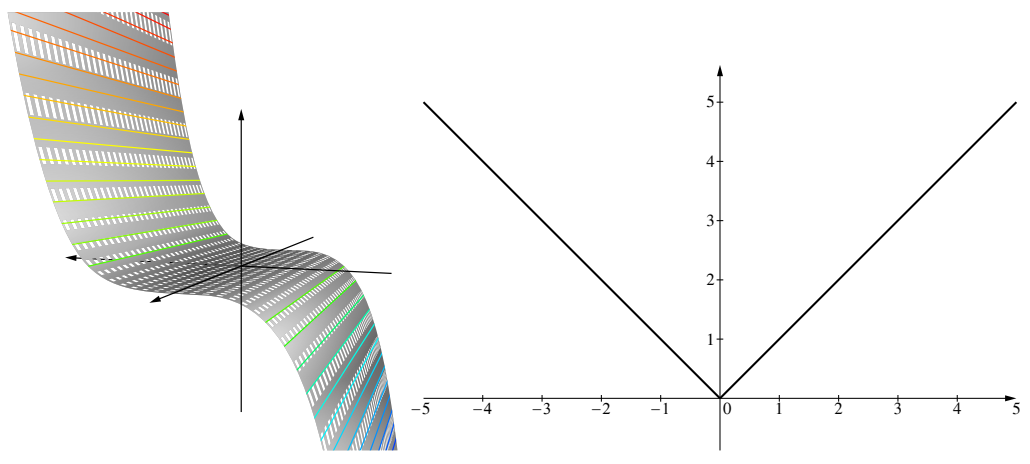

Figure 2. The graph of $f$ and the image of the curve $v$ comprising $u$.

Let us note that the rank-one assumption for $\mathrm{D} u$ is equivalent to the existence of two vector fields $\xi: \mathbb{R}^{n} \supseteq \Omega \rightarrow \mathbb{R}^{N}$ and $a: \mathbb{R}^{n} \supseteq \Omega \rightarrow \mathbb{R}^{n}$ such that $\mathrm{D} u=\xi \otimes a$ in $\Omega$.

Example 3 below shows that Theorem 2 is optimal and in general rank-one solutions to the system (1-1) can not have affine image but only piecewise affine.

Example 3. Consider the $C^{2}$ rank-one map $u: \mathbb{R}^{2} \rightarrow \mathbb{R}^{2}$ given by

$$
u(x, y)= \begin{cases}\left(-x^{4}, x^{4}\right), & x \leq 0, y \in \mathbb{R}, \\ \left(+x^{4}, x^{4}\right), & x>0, y \in \mathbb{R} .\end{cases}
$$

Then, $u=v \circ f$ with $v: \mathbb{R} \rightarrow \mathbb{R}^{2}$ given by $v(t)=(t,|t|)$ and $f: \mathbb{R}^{2} \rightarrow \mathbb{R}$ given by $f(x, y)=\operatorname{sgn}(x) x^{4}$ (see Figure 2).

It follows that $u$ solves (1-1) on $\mathbb{R}^{2}$ : indeed, $\Delta u$ is a nonvanishing vector field on $\{x \neq 0\}$, being tangential to the image thereon since it is parallel to the derivative $v^{\prime}(t)=(1, \pm 1)$ for $t \neq 0$. On the other hand, on $\{x=0\}$ we have that $\Delta u=0$. However, the image $u\left(\mathbb{R}^{2}\right)$ of $u$ is piecewise affine but not affine and equals $v(\mathbb{R})$. Note that (1-1) is underdetermined, especially without the requirement of boundary conditions. Therefore, the point of this example is to show that the solutions in general do not have affine image, although some of them may do, for instance the trivial affine ones.

As a consequence of Theorem 2, we obtain the next result regarding the rigidity of $p$-Harmonic maps for $p \in[2, \infty)$ which complements one of the results in the paper [17] wherein the case $p=\infty$ was considered.

Corollary 4 (rigidity of $p$-Harmonic maps, cf. [17]). Let $\Omega \subseteq \mathbb{R}^{n}$ be an open set and $n, N \geq 1$. Let $u \in C^{2}\left(\Omega, \mathbb{R}^{N}\right)$ be a $p$-Harmonic map in $\Omega$ for some $p \in[2, \infty)$, that is $u$ solves (1-3). Suppose that the rank of its gradient matrix is at most one:

$$
\operatorname{rk}(\mathrm{D} u) \leq 1 \quad \text { in } \Omega
$$

Then, the same result as in Theorem 2 is true.

In addition, there exists a partition of $\Omega$ to at most countably many Borel sets, where each set of the partition is a nonempty open set with a (perhaps empty) boundary portion, such that, on each of these, $u$ can be represented as

$$
u=v \circ f .
$$


Here, $f$ is a scalar $C^{2} p$-Harmonic function (for the respective $p \in[2, \infty)$ ), defined on an open neighbourhood of the Borel set, whilst $v: \mathbb{R} \rightarrow \mathbb{R}^{N}$ is a Lipschitz curve which is twice differentiable and with unit speed on the image of $f$.

Now we move on to discuss our second main result which concerns the rigidity of solutions

$$
u: \mathbb{R}^{2} \supseteq \Omega \rightarrow \mathbb{R}^{N}
$$

to (1-1) for $N \geq 2$, having the additively separated form

$$
u(x, y)=f(x)-f(y)
$$

for some curve $f: \mathbb{R} \rightarrow \mathbb{R}^{N}$. Solutions of this form are very important in relation to the $\infty$-Laplacian. If $N=1$, all $\infty$-Harmonic functions of this form after a normalisation reduce to the so-called Aronsson solution on $\mathbb{R}^{2}$

$$
u(x, y)=|x|^{4 / 3}-|y|^{4 / 3}
$$

which is the standard explicit example of a non- $C^{2} \infty$-Harmonic function with conjectured optimal regularity. In the vectorial case, the family of separated solutions is quite large. For $N=2$, a large class of such vectorial solutions was constructed in [16] and is given by

$$
u(x, y)=\int_{x}^{y}(\cos (K(t)), \sin (K(t))) \mathrm{d} t
$$

with $K$ a function in $C^{1}(\mathbb{R})$ satisfying certain general conditions. The simplest nontrivial example of an $\infty$-Harmonic map with this form (defined on the strip $\{|x-y|<\pi / 4\} \subseteq \mathbb{R}^{2}$ ) is given by the choice $K(t)=t$. Our second main result asserts that solutions of separated form to (1-1) have images which are piecewise affine, contained in a union of intersecting planes of $\mathbb{R}^{N}$. More precisely, we have:

Theorem 5 (rigidity and flatness of maps with tangential Laplacian in separated form). Let $\Omega \subseteq \mathbb{R}^{2}$ be an open set and let also $N \geq 2$. Let $u: \mathbb{R}^{n} \supseteq \Omega \rightarrow \mathbb{R}^{N}$ be a classical solution to the nonlinear system (1-1) in $\Omega$, having the separated form $u(x, y)=f(x)-f(y)$, for some curve $f \in\left(W^{3, p} \cap C^{2}\right)\left(\mathbb{R}, \mathbb{R}^{N}\right)$ and some $p>1$.

Then, the image $u(\Omega)$ of the solution is contained in an at most countable union of affine planes in $\mathbb{R}^{N}$.

In addition, the proof of Theorem 5 shows that every connected component of the set $\{\operatorname{rk}(\mathrm{D} u)=2\}$ is contained entirely in an affine plane and every connected component of the set $\{\operatorname{rk}(\mathrm{D} u) \leq 1\}$ is contained entirely in an affine line.

Note that our result is trivial in the case that $N=n=2$ since the codimension $N-n$ vanishes. Additionally, due to the regularity of the solutions, if a $C^{2}$ mapping has piecewise affine image, then second derivatives must vanish when first derivatives vanish at the "breaking points," namely the points where the image has a nonsmooth edge and no tangent exists. Further, one might also restrict their attention to domains of rectangular shape, since any map with separated form can be automatically extended to the smallest rectangle containing the domain.

Also, herein we consider only the illustrative case of $n=2<N$ and do not discuss more general situations, since numerical evidence obtained in [24] suggests that Theorem 5 does not hold in general for solutions in nonseparated form. 
In this paper we try to keep the exposition as simple as possible and therefore we refrain from discussing generalised solutions to (1-1) and (1-5) (or (1-3)). We confine ourselves to merely mentioning that in the scalar case, $\infty$-Harmonic functions are understood in the viscosity sense of Crandall-IshiiLions (see, e.g., [10;19]), whilst in the vectorial case a new candidate theory for systems has been proposed in [22] which has already borne significant fruit in $[20 ; 21 ; 22 ; 11 ; 9 ; 24]$.

We now expound on how exactly the nonlinear system (1-1) arises from (1-3) and (1-5). By expanding the derivatives in (1-3) and normalising, we arrive at

$$
\mathrm{D} u \otimes \mathrm{D} u: \mathrm{D}^{2} u+\frac{|\mathrm{D} u|^{2}}{p-2} \Delta u=0 .
$$

For any $X \in \mathbb{R}^{N \times n}$, let $\llbracket X \rrbracket^{\|}$denote the orthogonal projection on the range of the linear map $X: \mathbb{R}^{n} \rightarrow \mathbb{R}^{N}$ :

$$
\llbracket X \rrbracket^{\|}:=\operatorname{Proj}_{\mathrm{R}(X)} .
$$

Since the identity matrix of $\mathbb{R}^{N}$ splits as $\mathrm{I}=\llbracket \mathrm{D} u \rrbracket^{\|}+\llbracket \mathrm{D} u \rrbracket^{\perp}$, by expanding $\Delta u$ with respect to these projections, we have

$$
\mathrm{D} u \otimes \mathrm{D} u: \mathrm{D}^{2} u+\frac{|\mathrm{D} u|^{2}}{p-2} \llbracket \mathrm{D} u \rrbracket^{\|} \Delta u=-\frac{|\mathrm{D} u|^{2}}{p-2} \llbracket \mathrm{D} u \rrbracket^{\perp} \Delta u .
$$

The mutual perpendicularity of the vector fields of the left and right hand side leads via a renormalisation argument (see, e.g., $[15 ; 17 ; 18]$ ) to the equivalence of the $p$-Laplacian with the pair of systems

$$
\mathrm{D} u \otimes \mathrm{D} u: \mathrm{D}^{2} u+\frac{|\mathrm{D} u|^{2}}{p-2} \llbracket \mathrm{D} u \rrbracket^{\|} \Delta u=0, \quad|\mathrm{D} u|^{2} \llbracket \mathrm{D} u \rrbracket^{\perp} \Delta u=0 .
$$

The $\infty$-Laplacian corresponds to the limiting case of (1-10) as $p \rightarrow \infty$, which takes the form

$$
\mathrm{D} u \otimes \mathrm{D} u: \mathrm{D}^{2} u=0, \quad|\mathrm{D} u|^{2} \llbracket \mathrm{D} u \rrbracket^{\perp} \Delta u=0 .
$$

Hence, the $\infty$-Laplacian (1-5) actually consists of the two independent systems in (1-11) above. The system $|\mathrm{D} u|^{2} \llbracket \mathrm{D} u \rrbracket^{\perp} \Delta u=0$ is, at least on $\{\mathrm{D} u \neq 0\}$, equivalent to (1-1). Note that in the scalar case of $N=1$ as well as in the case of submersion solutions (for $N \leq n$ ), the second system trivialises.

We conclude the introduction with a geometric interpretation of the nonlinear system (1-1), which can be expressed in a more geometric language as follows: ${ }^{1}$ Suppose that $u(\Omega)$ is a $C^{2}$ manifold and let $\boldsymbol{A}(u)$ denote its second fundamental form. Then

$$
\llbracket \mathrm{D} u \rrbracket^{\perp} \Delta u=-\operatorname{tr} A(u)(\mathrm{D} u, \mathrm{D} u) .
$$

The tangential part $\llbracket \mathrm{D} u \rrbracket^{\|} \Delta u$ of the Laplacian is commonly called the tension field in the theory of Harmonic maps and is symbolised by $\tau(u)$ (see, e.g., [25]). Hence, we have the orthogonal decomposition

$$
\Delta u=\tau(u)-\operatorname{tr} A(u)(\mathrm{D} u, \mathrm{D} u) .
$$

\footnotetext{
${ }^{1}$ This fact has been brought to our attention by Roger Moser.
} 
Therefore, in the case of higher regularity of the image of $u$, we obtain that the nonlinear system

$$
\Delta u=\tau(u) \text { in } \Omega,
$$

is a further geometric reformulation of our PDE system (1-1).

\section{Proofs}

In this section we prove the results of this paper. Before delving into that, we present a result of independent interest in which we represent explicitly the vector field A arising in the parametric system $\Delta u=\mathrm{D} u \mathrm{~A}$, in the illustrative case of $n=2$.

We will be using the symbolisations "cof," "det" and "rk" to denote the cofactor matrix, the determinant function and the rank of a matrix, respectively.

Lemma 6 (representation of A). Let $u \in C^{2}\left(\Omega, \mathbb{R}^{N}\right)$ be given, $\Omega \subseteq \mathbb{R}^{2}$ open, $N \geq 2$. The following are equivalent:

(1) The map $u$ is a solution to the PDE system (1-1).

(2) There exists a vector field $\mathrm{A}: \mathbb{R}^{2} \supseteq \Omega \rightarrow \mathbb{R}^{N}$ such that

$$
\Delta u=\mathrm{D} u \mathrm{~A} \text { in } \Omega \text {. }
$$

In (2), as A one might choose

$$
\overline{\mathrm{A}}:= \begin{cases}\frac{\operatorname{cof}\left(\mathrm{D} u^{\top} \mathrm{D} u\right)^{\top}}{\operatorname{det}\left(\mathrm{D} u^{\top} \mathrm{D} u\right)}(\mathrm{D} u)^{\top} \Delta u & \text { on }\{\operatorname{rk}(\mathrm{D} u)=2\} \\ (\Delta u)^{\top} \frac{\mathrm{D} u \mathrm{D} u^{\top}}{\left|\mathrm{D} u \mathrm{D} u^{\top}\right|^{2}} \mathrm{D} u & \text { on }\{\operatorname{rk}(\mathrm{D} u)=1\}, \\ 0 & \text { on }\{\operatorname{rk}(\mathrm{D} u)=0\} .\end{cases}
$$

A is uniquely determined on $\{\operatorname{rk}(\mathrm{D} u)=2\}$ but not on $\{\operatorname{rk}(\mathrm{D} u)<2\}$ and any other $\mathrm{A}$ has the form $\overline{\mathrm{A}}+V$, where $V(x)$ lies in the nullspace of $\mathrm{D} u(x), x \in \Omega$.

Proof of Lemma 6. The equivalence between (1) and (2) is immediate, therefore it suffices to show that $\overline{\mathrm{A}}$ satisfies $\Delta u=\mathrm{D} u \overline{\mathrm{A}}$ and is unique on $\{\operatorname{rk}(\mathrm{D} u)=2\}$. Let $\mathrm{A}$ be as in $(2)$. On $\{\operatorname{rk}(\mathrm{D} u)=2\}$, the $2 \times 2$ matrix-valued map $\mathrm{D} u^{\top} \mathrm{D} u$ is invertible and

$$
\left(\mathrm{D} u^{\top} \mathrm{D} u\right)^{-1}=\frac{\operatorname{cof}\left(\mathrm{D} u^{\top} \mathrm{D} u\right)^{\top}}{\operatorname{det}\left(\mathrm{D} u^{\top} \mathrm{D} u\right)}
$$

Since $\mathrm{D} u^{\top} \Delta u=\mathrm{D} u^{\top} \mathrm{D} u \mathrm{~A}$, we obtain that $\mathrm{A}=\overline{\mathrm{A}}$.

The claim being obvious for $\{\operatorname{rk}(\mathrm{D} u)=0\}=\{\mathrm{D} u=0\}$, it suffices to consider only the set $\{\operatorname{rk}(\mathrm{D} u)=1\}$ in order to conclude. Thereon we have that $\mathrm{D} u$ can be written as

$$
\mathrm{D} u=\xi \otimes a, \quad \text { in }\{\operatorname{rk}(\mathrm{D} u)=1\},
$$

for some nonvanishing vector fields $\xi$ and $a$. By replacing $\xi$ with $\xi|a|$ and $a$ with $a /|a|$, we may assume $|a| \equiv 1$ throughout $\{\operatorname{rk}(\mathrm{D} u)=1\}$. If $\Delta u=\mathrm{D} u \mathrm{~A}$, we have $\Delta u=(\xi \otimes a) \mathrm{A}$ and since any component of A which is orthogonal to $a$ is annihilated, we may replace A by $\lambda a$ for some function $\lambda$. Therefore,

$$
\Delta u=(\xi \otimes a) \mathrm{A}=(\xi \otimes a)(\lambda a)=\xi \lambda|a|^{2}=\lambda \xi
$$


and hence $\xi \cdot \Delta u=\lambda|\xi|^{2}$ and also $\xi^{\top} \mathrm{D} u=a|\xi|^{2}$. On the other hand, since

$$
\mathrm{D} u \mathrm{D} u^{\top}=(\xi \otimes a)(a \otimes \xi)=\xi \otimes \xi, \quad\left|\mathrm{D} u \mathrm{D} u^{\top}\right|=|\xi|^{2}
$$

we infer that

$$
\begin{aligned}
\mathrm{A} & =\lambda a=\left(\frac{\Delta u \cdot \xi}{\left|\xi^{2}\right|}\right)\left(\frac{\xi^{\top} \mathrm{D} u}{\left|\xi^{2}\right|}\right)=\frac{\Delta u^{\top}(\xi \otimes \xi) \mathrm{D} u}{|\xi \otimes \xi|^{2}} \\
& =(\Delta u)^{\top} \frac{\mathrm{D} u \mathrm{D} u^{\top}}{\left|\mathrm{D} u \mathrm{D} u^{\top}\right|^{2}} \mathrm{D} u
\end{aligned}
$$

as claimed.

We now continue with the proof of the main results.

The main analytical tool needed in the proof of Theorem 2 is the next rigidity theorem for maps whose gradient has rank at most one. It was established in [17] and we recall it below for the convenience of the reader and only in the case needed in this paper.

Theorem 7 (rigidity of rank-one maps, cf. [17]). Suppose $\Omega \subseteq \mathbb{R}^{n}$ is an open set and $u$ is in $C^{2}\left(\Omega, \mathbb{R}^{N}\right)$. Then, the following are equivalent:

(i) The map $u$ satisfies that $\operatorname{rk}(D u) \leq 1$ on $\Omega$. Equivalently, there exist vector fields $\xi: \Omega \rightarrow \mathbb{R}^{N}$ and $a: \Omega \rightarrow \mathbb{R}^{n}$ with $a \in C^{1}\left(\Omega, \mathbb{R}^{n}\right)$ and $\xi \in C^{1}\left(\Omega \backslash\{a=0\}, \mathbb{R}^{N}\right)$ such that

$$
\mathrm{D} u=\xi \otimes a, \quad \text { on } \Omega .
$$

(ii) There exists Borel subset $\left\{B_{i}\right\}_{i \in \mathbb{N}}$ of $\Omega$ such that

$$
\Omega=\bigcup_{i=1}^{\infty} B_{i}
$$

and each $B_{i}$ equals a nonempty connected open set with a (possibly empty) boundary portion, functions $\left\{f_{i}\right\}_{i \in \mathbb{N}} \in C^{2}(\Omega)$ and curves $\left\{v_{i}\right\}_{i \in \mathbb{N}} \subseteq W_{\text {loc }}^{1, \infty}\left(\mathbb{R}, \mathbb{R}^{N}\right)$ such that, on each $B_{i}$ the map $u$ has the form

$$
u=v_{i} \circ f_{i}, \quad \text { on } B_{i} .
$$

Moreover, $\left|v_{i}^{\prime}\right| \equiv 1$ on the interval $f_{i}\left(B_{i}\right), v_{i}^{\prime} \equiv 0$ on $\mathbb{R} \backslash f_{i}\left(B_{i}\right)$ and $v_{i}^{\prime \prime}$ exists everywhere on $f_{i}\left(B_{i}\right)$, interpreted as 1-sided derivative on $\partial f_{i}\left(B_{i}\right)$ (if $f_{i}\left(B_{i}\right)$ is not open). Also,

$$
\left\{\begin{aligned}
\mathrm{D} u & =\left(v_{i}^{\prime} \circ f_{i}\right) \otimes \mathrm{D} f_{i} & & \text { on } B_{i}, \\
\mathrm{D}^{2} u & =\left(v_{i}^{\prime \prime} \circ f_{i}\right) \otimes \mathrm{D} f_{i} \otimes \mathrm{D} f_{i}+\left(v_{i}^{\prime} \circ f_{i}\right) \otimes \mathrm{D}^{2} f_{i} & & \text { on } B_{i} .
\end{aligned}\right.
$$

In addition, the local functions $\left(f_{i}\right)_{1}^{\infty}$ extend to a global function $f \in C^{2}(\Omega)$ with the same properties as above if $\Omega$ is contractible (namely, homotopically equivalent to a point).

We may now prove our first main result.

Proof of Theorem 2. Suppose that $u: \mathbb{R}^{n} \supseteq \Omega \rightarrow \mathbb{R}^{N}$ is a solution to the nonlinear system (1-1) in $C^{2}\left(\Omega, \mathbb{R}^{N}\right)$ which in addition satisfies that $\operatorname{rk}(\mathrm{D} u) \leq 1$ in $\Omega$. Since $\{\mathrm{D} u=0\}$ is closed, necessarily its complement in $\Omega$ which is $\{\operatorname{rk}(\mathrm{D} u)=1\}$ is open. 
By invoking Theorem 7, we have that there exists a partition of the open subset $\{\operatorname{rk}(\mathrm{D} u)=1\}$ to countably many Borel sets $\left(B_{i}\right)_{1}^{\infty}$ with respective functions $\left(f_{i}\right)_{1}^{\infty}$ and curves $\left(v_{i}\right)_{1}^{\infty}$ as in the statement such that (2-1)-(2-2) hold true and in addition

$$
\mathrm{D} f_{i} \neq 0 \quad \text { on } B_{i}, i \in \mathbb{N} \text {. }
$$

Consequently, on each $B_{i}$ we have

$$
\begin{aligned}
\llbracket \mathrm{D} u \rrbracket^{\perp} & =\llbracket\left(v_{i}^{\prime} \circ f_{i}\right) \otimes \mathrm{D} f_{i} \rrbracket^{\perp}=\mathrm{I}-\frac{\left(v_{i}^{\prime} \circ f_{i}\right) \otimes\left(v_{i}^{\prime} \circ f_{i}\right)}{\left|v_{i}^{\prime} \circ f_{i}\right|^{2}}, \\
\Delta u & =\left(v_{i}^{\prime \prime} \circ f_{i}\right)\left|\mathrm{D} f_{i}\right|^{2}+\left(v_{i}^{\prime} \circ f_{i}\right) \Delta f_{i} .
\end{aligned}
$$

Hence, (1-1) becomes

$$
\left[\mathrm{I}-\frac{\left(v_{i}^{\prime} \circ f_{i}\right) \otimes\left(v_{i}^{\prime} \circ f_{i}\right)}{\left|v_{i}^{\prime} \circ f_{i}\right|^{2}}\right]\left(\left(v_{i}^{\prime \prime} \circ f_{i}\right)\left|\mathrm{D} f_{i}\right|^{2}+\left(v_{i}^{\prime} \circ f_{i}\right) \Delta f_{i}\right)=0,
$$

on $B_{i}$. Since $\left|v_{i}\right|^{2} \equiv 1$ on $f_{i}\left(B_{i}\right)$, we have that $v_{i}^{\prime \prime}$ is orthogonal to $v_{i}^{\prime}$ thereon and therefore the above equation reduces to

$$
\left(v_{i}^{\prime \prime} \circ f_{i}\right)\left|\mathrm{D} f_{i}\right|^{2}=0 \quad \text { on } B_{i}, i \in \mathbb{N} .
$$

Therefore, $v_{i}$ is affine on the interval $f_{i}\left(B_{i}\right) \subseteq \mathbb{R}$ and as a result $u\left(B_{i}\right)=v_{i}\left(f_{i}\left(B_{i}\right)\right)$ is contained in an affine line of $\mathbb{R}^{N}$, for each $i \in \mathbb{N}$. On the other hand, since

$$
u(\Omega)=u(\{\mathrm{D} u=0\}) \bigcup_{i \in \mathbb{N}} u\left(B_{i}\right)
$$

and $u$ is constant on each connected component of the interior of $\{\mathrm{D} u=0\}$, the conclusion ensues by the regularity of $u$ because $u(\{\mathrm{D} u=0\})$ is also contained in the previous union of affine lines. The result ensues.

Now we establish Corollary 4 by following similar lines to those of the respective result in [17].

Proof of Corollary 4. Suppose $u$ is as in the statement of the corollary. By Theorem 7, there exists, a partition of $\Omega$ to Borel sets $\left\{B_{i}\right\}_{i \in \mathbb{N}}$, functions $f_{i} \in C^{2}(\Omega)$ and Lipschitz curves $\left\{v_{i}\right\}_{i \in \mathbb{N}}: \mathbb{R} \rightarrow \mathbb{R}^{N}$ with $\left|v_{i}^{\prime}\right| \equiv 1$ on $f_{i}\left(B_{i}\right),\left|v_{i}^{\prime}\right| \equiv 0$ on $\mathbb{R} \backslash f_{i}\left(B_{i}\right)$ and twice differentiable on $f_{i}\left(B_{i}\right)$, such that $\left.u\right|_{B_{i}}=v_{i} \circ f_{i}$ and (2-2) holds as well. Since on each $B_{i}$ we have

$$
|\mathrm{D} u|=\left|\left(v_{i}^{\prime} \circ f_{i}\right) \otimes \mathrm{D} f_{i}\right|=\left|\mathrm{D} f_{i}\right|,
$$

by (1-8) and the above, we obtain

$$
\begin{aligned}
& \left(\left(v_{i}^{\prime} \circ f_{i}\right) \otimes \mathrm{D} f_{i}\right) \otimes\left(\left(v_{i}^{\prime} \circ f_{i}\right) \otimes \mathrm{D} f\right): \\
& \quad\left[\left(v_{i}^{\prime \prime} \circ f_{i}\right) \otimes \mathrm{D} f_{i} \otimes \mathrm{D} f_{i}+\left(v_{i}^{\prime} \circ f_{i}\right) \otimes \mathrm{D}^{2} f_{i}\right]+\frac{\left|\mathrm{D} f_{i}\right|^{2}}{p-2}\left\{\left(v_{i}^{\prime} \circ f_{i}\right) \Delta f_{i}+\left(v_{i}^{\prime \prime} \circ f\right)\left|\mathrm{D} f_{i}\right|^{2}\right\}=0,
\end{aligned}
$$

on $B_{i}$. Since $v_{i}^{\prime \prime}$ is orthogonal to $v_{i}^{\prime}$ and also $v_{i}^{\prime}$ has unit length, the above reduces to

$$
\left(v_{i}^{\prime} \circ f_{i}\right)\left[\mathrm{D} f_{i} \otimes D f_{i}: \mathrm{D}^{2} f_{i}+\frac{\left|\mathrm{D} f_{i}\right|^{2}}{p-2} \Delta f_{i}\right]+\frac{1}{p-2}\left(v_{i}^{\prime \prime} \circ f_{i}\right)\left|\mathrm{D} f_{i}\right|^{4}=0,
$$


on $B_{i}$. Again by orthogonality, the above is equivalent to the pair of independent systems

$$
\left(v_{i}^{\prime} \circ f_{i}\right)\left[\mathrm{D} f_{i} \otimes D f_{i}: \mathrm{D}^{2} f_{i}+\frac{\left|\mathrm{D} f_{i}\right|^{2}}{p-2} \Delta f_{i}\right]=0, \quad\left(v_{i}^{\prime \prime} \circ f_{i}\right)\left|\mathrm{D} f_{i}\right|^{4}=0,
$$

on $B_{i}$. Since $\left|v_{i}^{\prime}\right| \equiv 1$ of $f_{i}\left(B_{i}\right)$, it follows that $\Delta_{p} f_{i}=0$ on $B_{i}$ and since $\left(B_{i}\right)_{1}^{\infty}$ is a partition of $\Omega$ of the form described in the statement, the result ensues by invoking Theorem 2.

We may now prove our second main result.

Proof of Theorem 5. The system $\llbracket \mathrm{D} u \rrbracket^{\perp} \Delta u=0$ is equivalent to

$$
\Delta u=\mathrm{D} u \cdot A
$$

for a vector field $A$ with components $a, b$. Then (2-3) can be rewritten as

$$
f^{\prime \prime}(x)-f^{\prime \prime}(y)=a(x, y) f^{\prime}(x)-b(x, y) f^{\prime}(y) .
$$

The choices $(x, y)=(z, z+t)$ and $(x, y)=(z+t, z)$ in (2-4) yield the equations

$$
f^{\prime \prime}(z)-f^{\prime \prime}(z+t)=a(z, z+t) f^{\prime}(z)-b(z, z+t) f^{\prime}(z+t)
$$

and

$$
f^{\prime \prime}(z+t)-f^{\prime \prime}(z)=a(z+t, z) f^{\prime}(z+t)-b(z+t, z) f^{\prime}(z)
$$

respectively. Let $f_{\alpha}$ denote the $\alpha$-component of $f, \alpha=1, \ldots, N$. By subtracting (2-5) from (2-6) we get for $t \neq 0$ that

$$
\begin{aligned}
2 \frac{f_{\alpha}^{\prime \prime}(z+t)-f_{\alpha}^{\prime \prime}(z)}{t}=(a(z+t, z) & +b(z, z+t)) \frac{f_{\alpha}^{\prime}(z+t)-f_{\alpha}^{\prime}(z)}{t} \\
& +f_{\alpha}^{\prime}(z)\left(\frac{a(z+t, z)-a(z, z+t)}{t}+\frac{b(z, z+t)-b(z+t, z)}{t}\right)
\end{aligned}
$$

for $\alpha=1, \ldots, N$. On the set $\left\{f_{\alpha}^{\prime}=0\right\}$, Equation (2-7) becomes

$$
2 f_{\alpha}^{\prime \prime \prime}(z)=(\bar{a}(z, z)+\bar{b}(z, z)) f_{\alpha}^{\prime \prime}(z)
$$

as $t \rightarrow 0$. Note also that $\left\{f_{\alpha}^{\prime}=0\right\}$ is closed and its complement $\left\{f_{\alpha}^{\prime} \neq 0\right\}$ is open. Now let us set

$$
C_{\alpha}(z, t):=\frac{a(z+t, z)-a(z, z+t)}{t}+\frac{b(z, z+t)-b(z+t, z)}{t} .
$$

On $\left\{f_{\alpha}^{\prime} \neq 0\right\},(2-7)$ yields that

$$
C_{\alpha}(z, t)=\frac{1}{f_{\alpha}^{\prime}(z)}\left[2 \frac{f_{\alpha}^{\prime \prime}(z+t)-f_{\alpha}^{\prime \prime}(z)}{t}-(a(z+t, z)+b(z, z+t)) \frac{f_{\alpha}^{\prime}(z+t)-f_{\alpha}^{\prime}(z)}{t}\right] .
$$

Fix an index $\alpha \in\{1, \ldots, N\}, \delta>0$, an infinitesimal sequence $\left(t_{m}\right)_{1}^{\infty}$ and consider the inner $\delta$-neighbourhood $\mathcal{O}_{\delta}$ of the set $\left\{f_{\alpha}^{\prime} \neq 0\right\}$, namely

$$
\mathcal{O}_{\delta}:=\left\{x \in \mathbb{R}^{n}: f_{\alpha}^{\prime}(x) \neq 0 \text { and } \operatorname{dist}\left(x, \partial\left\{f_{\alpha}^{\prime} \neq 0\right\}\right)>\delta\right\}
$$


Then for any fixed $\delta>0$ small, there exists a constant $c_{\delta}>0$ such that along the sequence $t_{m} \rightarrow 0$ we have

$$
\begin{aligned}
\left\|C_{\alpha}\left(\cdot, t_{m}\right)\right\|_{L^{p}\left(\mathcal{O}_{\delta}\right)} & \leq 2\left\|\frac{1}{f_{\alpha}^{\prime}(\cdot)} \frac{f_{\alpha}^{\prime \prime}\left(\cdot+t_{m}\right)-f_{\alpha}^{\prime \prime}(\cdot)}{t_{m}}\right\|_{L^{p}\left(\mathcal{O}_{\delta}\right)} \quad+\|a+b\|_{L^{\infty}(\Omega)}\left\|\frac{1}{f_{\alpha}^{\prime}(\cdot)} \frac{f_{\alpha}^{\prime}\left(\cdot+t_{m}\right)-f_{\alpha}^{\prime}(\cdot)}{t_{m}}\right\|_{L^{p}\left(\mathcal{O}_{\delta}\right)} \\
& \leq \frac{1}{c_{\delta}}\left(2\left\|f_{\alpha}^{\prime \prime \prime}\right\|_{L^{p}\left(\mathcal{O}_{\delta}\right)}+\|a+b\|_{L^{\infty}(\Omega)}\left\|f_{\alpha}^{\prime \prime}\right\|_{L^{p}\left(\mathcal{O}_{\delta}\right)}\right) \\
& \leq \frac{1}{c_{\delta}}\left(2\left\|f^{\prime \prime \prime}\right\|_{L^{p}(\mathbb{R})}+\|a+b\|_{L^{\infty}(\Omega)}\left\|f^{\prime \prime}\right\|_{L^{p}(\mathbb{R})}\right) .
\end{aligned}
$$

Note that the right hand side of the above estimate is bounded uniformly in $m \in \mathbb{N}$ as $f^{\prime \prime \prime} \in L^{p}\left(\mathbb{R}, \mathbb{R}^{N}\right)$ and $f^{\prime} \in C^{1}\left(\mathbb{R}, \mathbb{R}^{N}\right)$. By letting $\delta \rightarrow 0$ and using a standard diagonal argument, (2-9) implies that there exists a function $\bar{C}_{\alpha}$ such that

$$
C_{\alpha}\left(\cdot, t_{m}\right) \rightarrow \bar{C}_{\alpha} \quad \text { in } L_{\mathrm{loc}}^{p}\left(\left\{f_{\alpha}^{\prime} \neq 0\right\}\right),
$$

as $m \rightarrow \infty$ along a subsequence of indices $\left(m_{k}\right)_{1}^{\infty}$. As a result, (2-7) becomes

$$
2 f_{\alpha}^{\prime \prime \prime}(z)=(\bar{a}(z, z)+\bar{b}(z, z)) f_{\alpha}^{\prime \prime}(z)+f_{\alpha}^{\prime}(z) \bar{C}_{\alpha}(z) \quad \text { on }\left\{f_{\alpha}^{\prime} \neq 0\right\}
$$

for any $\alpha=1, \ldots, N$. Combining equations (2-8) and (2-10), we infer that there exist measurable functions $A, B: \mathbb{R} \rightarrow \mathbb{R}$ such that

$$
f^{\prime \prime \prime}=A f^{\prime}+B f^{\prime \prime} \quad \text { a.e. on } \mathbb{R} .
$$

The goal in now to show that (2-11) implies that the torsion of the curve $f$ vanishes, at least on a union of subintervals of $\mathbb{R}$. The idea is to project on three-dimensional subspaces of $\mathbb{R}^{N}$ in order to utilise standard ideas of elementary differential geometry of curves.

To this end, let $P_{3}: \mathbb{R}^{N} \rightarrow \mathbb{R}^{N}$ be the orthogonal projection on a $3 D$ subspace $V_{3} \equiv P_{3}\left(\mathbb{R}^{N}\right)$ of $\mathbb{R}^{N}$. The choice of 3-dimensional subspaces owes to the fact that we would like to use the classical formulas of differential geometry of curves in the Euclidean space. Then, $P_{3} f: \mathbb{R} \rightarrow V_{3} \cong \mathbb{R}^{3}$ is a curve in $\mathbb{R}^{3}$, which is $C^{2}$. By (2-11) we have,

$$
\left(P_{3} f\right)^{\prime \prime \prime}=A\left(P_{3} f\right)^{\prime}+B\left(P_{3} f\right)^{\prime \prime} \quad \text { a.e. on } \mathbb{R} .
$$

Let " $\times$ " denote the cross (exterior) product in $\mathbb{R}^{3}$. Then, the curvature of $P_{3} f$ is given by

$$
\kappa=\left|\left(P_{3} f\right)^{\prime} \times\left(P_{3} f\right)^{\prime \prime}\right|
$$

and, on $\{\kappa \neq 0\}$, the torsion is given by

$$
\tau=\frac{\left[\left(P_{3} f\right)^{\prime} \times\left(P_{3} f\right)^{\prime \prime}\right] \cdot\left(P_{3} f\right)^{\prime \prime \prime}}{\left|\left(P_{3} f\right)^{\prime} \times\left(P_{3} f\right)^{\prime \prime}\right|^{2}} .
$$

Note that $\{\kappa \neq 0\}$ is open, as $P_{3} f$ is $C^{2}$. Then, we have:

- On the topological interior $\operatorname{int}(\{\kappa=0\}), P_{3} f$ is contained in an affine line of $V_{3}$.

- On the topological interior $\operatorname{int}(\{\kappa \neq 0\}), P_{3} f$ is planar and hence contained in affine plane of $V_{3}$. 
Since $\partial(\{\kappa=0\})$ is a nowhere dense set (as the boundary of a closed set which contains no open set), it follows that $f(\partial(\{\kappa=0\}))$ is contained in the boundary of an affine plane or an affine line. Hence, we have that, for any projection $P_{3} f$ on a 3-dimensional subspace of $\mathbb{R}^{N}$, the projected curve is contained in an at most countable union of affine planes and lines. Therefore, the same is true for $f$ itself by elementary analytic geometry: if all 3-dimensional projections of the image set in the space $\mathbb{R}^{N}$ for $n \geq 3$ are planes or lines, the same is true for the image itself. The conclusion follows.

\section{Acknowledgements}

Katzourakis is indebted to G. Aronsson for his encouragement and support towards him. He would also like to thank Roger Moser and Giles Shaw for their comments and suggestions which improved the content of this paper, as well as Jan Kristensen, Tristan Pryer and Enea Parini for several scientific discussions relevant to the results herein. Finally, all authors would like to thank the anonymous referee of the paper for their constructive comments and critique.

\section{References}

[1] H. Abugirda and N. Katzourakis, "Existence of $1 D$ vectorial absolute minimisers in $L^{\infty}$ under minimal assumptions", Proc. Amer. Math. Soc. 145:6 (2017), 2567-2575.

[2] G. Aronsson, "Minimization problems for the functional $\sup _{x} F\left(x, f(x), f^{\prime}(x)\right)$ ", Ark. Mat. 6 (1965), $33-53$.

[3] G. Aronsson, "Minimization problems for the functional $\sup _{x} F\left(x, f(x), f^{\prime}(x)\right)$, II", Ark. Mat. 6 (1966), 409-431.

[4] G. Aronsson, "Extension of functions satisfying Lipschitz conditions”, Ark. Mat. 6 (1967), 551-561.

[5] G. Aronsson, "On the partial differential equation $u_{x}^{2} u_{x x}+2 u_{x} u_{y} u_{x y}+u_{y}^{2} u_{y y}=0$ ", Ark. Mat. 7 (1968), 395-425.

[6] G. Aronsson, "Minimization problems for the functional $\sup _{x} F\left(x, f(x), f^{\prime}(x)\right)$, III", Ark. Mat. 7 (1969), 509-512.

[7] G. Aronsson, "On certain singular solutions of the partial differential equation $u_{x}^{2} u_{x x}+2 u_{x} u_{y} u_{x y}+u_{y}^{2} u_{y y}=0$ ", Manuscripta Math. 47:1-3 (1984), 133-151.

[8] G. Aronsson, "Construction of singular solutions to the $p$-harmonic equation and its limit equation for $p=\infty$ ", Manuscripta Math. 56:2 (1986), 135-158.

[9] B. Ayanbayev and N. Katzourakis, "A pointwise characterisation of the PDE system of vectorial calculus of variations in $L^{\infty}$, Proc. Roy. Soc. Edinburgh Sect. A (online publication February 2019).

[10] M. G. Crandall, "A visit with the $\infty$-Laplace equation”, pp. 75-122 in Calculus of variations and nonlinear partial differential equations, edited by B. Dacorogna and P. Marcellini, Lecture Notes in Math. 1927, Springer, 2008.

[11] G. Croce, N. Katzourakis, and G. Pisante, "DD-solutions to the system of vectorial calculus of variations in $L^{\infty}$ via the singular value problem”, Discrete Contin. Dyn. Syst. 37:12 (2017), 6165-6181.

[12] B. Dacorogna, Direct methods in the calculus of variations, 2nd ed., Applied Mathematical Sciences 78, Springer, 2008.

[13] A. Elmoataz, M. Toutain, and D. Tenbrinck, "On the $p$-Laplacian and $\infty$-Laplacian on graphs with applications in image and data processing”, SIAM J. Imaging Sci. 8:4 (2015), 2412-2451.

[14] L. C. Evans, Partial differential equations, 2nd ed., Graduate Studies in Mathematics 19, Amer. Math. Soc., Providence, RI, 2010.

[15] N. I. Katzourakis, " $L^{\infty}$ variational problems for maps and the Aronsson PDE system”, J. Differential Equations 253:7 (2012), 2123-2139.

[16] N. Katzourakis, "Explicit 2D $\infty$-harmonic maps whose interfaces have junctions and corners", C. R. Math. Acad. Sci. Paris 351:17-18 (2013), 677-680.

[17] N. Katzourakis, “On the structure of $\infty$-harmonic maps”, Comm. Partial Differential Equations 39:11 (2014), 2091-2124. 
[18] N. I. Katzourakis, “ $\infty$-minimal submanifolds”, Proc. Amer. Math. Soc. 142:8 (2014), 2797-2811.

[19] N. Katzourakis, An introduction to viscosity solutions for fully nonlinear PDE with applications to calculus of variations in $L^{\infty}$, Springer, 2015.

[20] N. Katzourakis, "Absolutely minimising generalised solutions to the equations of vectorial calculus of variations in $L^{\infty}$ ", Calc. Var. Partial Differential Equations 56:1 (2017), Paper No. 15, 25.

[21] N. Katzourakis, "A characterisation of $\infty$-harmonic and $p$-harmonic maps via affine variations in $L^{\infty}$ ", Electron. J. Differential Equations (2017), Paper No. 29, 19.

[22] N. Katzourakis, "Generalised solutions for fully nonlinear PDE systems and existence-uniqueness theorems", J. Differential Equations 263:1 (2017), 641-686.

[23] N. Katzourakis, "Inverse optical tomography through PDE constrained optimization $L^{\infty}$ ", SIAM J. Control Optim. 57:6 (2019), 4205-4233.

[24] N. Katzourakis and T. Pryer, "On the numerical approximation of $\infty$-harmonic mappings", NoDEA Nonlinear Differential Equations Appl. 23:6 (2016), Art. 51, 23.

[25] R. Moser, “An $L^{p}$ regularity theory for harmonic maps", Trans. Amer. Math. Soc. 367:1 (2015), 1-30.

HUSSIEN ABUGIRDA: hussien.abugirda@uobasrah.edu.iq

Department of Mathematics, College of Science, University of Basra, Basra, Iraq

and

Department of Mathematics and Statistics, University of Reading, Whiteknight, Reading, United Kingdom hussien.abugirda@reading.ac.uk

BIRZHAN AYANBAYEV: b.ayanbayev@pgr.reading.ac.uk

Department of Mathematics and Statistics, University of Reading, Reading, United Kingdom

NiKOS KATZOURAKIS: n.katzourakis@ reading.ac.uk

Department of Mathematics and Statistics, University of Reading, Reading, United Kingdom

RMJ - prepared by for the

Rocky Mountain Mathematics Consortium 